\title{
Comparison of clinical manifestations and treatment outcome according to age groups in adult patients with miliary tuberculosis
}

\author{
Jaehee Lee ${ }^{1 \#}$, Jae Kwang Lim" ${ }^{2 \#}$ Eun Jin Kim³, Deok Heon Lee ${ }^{4}$, Yu Kyung Kim ${ }^{5}$, Seung Soo Yoo ${ }^{1}$, Shin \\ Yup Lee ${ }^{1}$, Seung Ick Cha ${ }^{1}$, Jae Yong Park ${ }^{1}$, Chang Ho Kim ${ }^{1}$ \\ ${ }^{1}$ Department of Internal Medicine, ${ }^{2}$ Department of Radiology, School of Medicine, Kyungpook National University, Daegu, Republic of Korea; \\ ${ }^{3}$ Department of Internal Medicine, Daegu Catholic University Medical Center, Daegu, Republic of Korea; ${ }^{4}$ Department of Thoracic and \\ Cardiovascular Surgery, ${ }^{5}$ Department of Clinical Pathology, School of Medicine, Kyungpook National University, Daegu, Republic of Korea \\ Contributions: (I) Conception and design: J Lee, JK Lim, CH Kim; (II) Administrative support: None; (III) Provision of study materials or patients: \\ None; (IV) Collection and assembly of data: All authors; (V) Data analysis and interpretation: J Lee, JK Lim, CH Kim; (VI) Manuscript writing: All \\ authors; (VII) Final approval of manuscript: All authors. \\ \#These authors contributed equally to this work. \\ Correspondence to: Chang Ho Kim. Department of Internal Medicine, Kyungpook National University, School of Medicine, 680 Gukchaebosang-ro, \\ Jung-gu, Daegu, 700-842, Republic of Korea. E-mail: kimch@knu.ac.kr.
}

Background: After the introduction of chemotherapy, miliary tuberculosis (TB) has been more prevalent in adults than in children. However, adult patients have a wide age range and may have different characteristics across the age span. Thus, clinical manifestations and treatment outcome may differ according to age groups in adult patients with miliary TB. However, there is limited information regarding this issue.

Methods: Adult patients with miliary TB were retrospectively reviewed and were categorized into young (18-40 years), middle-aged (41-64 years), and old adults ( $\geq 65$ years). The clinical manifestations and treatment outcome were compared among the three adult groups.

Results: Of 150 patients, 27, 35, and 88 patients composed the young, middle-aged, and old adult groups, respectively. Overall clinical manifestations were comparable among the three groups. Treatment completion was significantly lower and overall TB deaths were significantly higher in the old group than in the young group. However, deaths in the young and middle-aged groups were all TB-related deaths, whereas deaths in the old group were more attributable to TB-unrelated deaths rather than TB-related deaths. In multivariate analysis, underlying chronic condition, lower hemoglobin levels, and acute respiratory failure were independent predictors for TB-related deaths in the adult group $<65$ years, and lower albumin levels and acute respiratory failure were those in the adult group $\geq 65$ years.

Conclusions: The present study suggests that treatment completion, the cause of death, and risk factors for TB-related deaths may be different according to age groups in adult patients with miliary TB.

Keywords: Miliary tuberculosis; treatment outcome; cause of death; risk factors for mortality

Submitted Dec 24, 2017. Accepted for publication Apr 18, 2018.

doi: $10.21037 /$ jtd.2018.04.139

View this article at: http://dx.doi.org/10.21037/jtd.2018.04.139

\section{Introduction}

Miliary tuberculosis (TB) results from massive lymphohematogenous dissemination of Mycobacterium tuberculosis (MTB) during both primary and post-primary TB $(1,2)$. After the introduction of chemotherapy, this disease has been more prevalent in middle-aged and older individuals than in children and young adults (3-5). With advances in medicine, anti-TB drugs since the 1970s are more effective and early diagnosis of miliary TB is more feasible by chest computed tomography (CT) (6-8). In contrast, elderly population with underlying diseases, who 
is more susceptible to TB or death, is gradually increasing.

The majority of previous studies have provided the overall clinical characteristics of adult patients with miliary TB (3-5,9-19). However, the adult patients have a wide age range and may have different characteristics across the age range. It may account for the variable mortality rate (3-50\%) in this disease (9-19). Thus, the clinical manifestations, treatment outcome, and cause of death in adult patients with miliary TB may differ according to the age range. However, there is limited information regarding this issue.

The present study compared the clinical manifestations, treatment outcome, and cause of death according to age groups in adult patients with miliary TB.

\section{Methods}

\section{Patients and study design}

The study was retrospectively conducted at Kyungpook National University Hospital, a tertiary referral hospital in South Korea, which is an area where active TB is a major health problem, with an intermediate prevalence of 101 cases per 100,000 persons, an incidence of 86 cases per 100,000 persons, and a mortality rate of 3.8 cases per 100,000 persons reported in 2014 (20). The Bacillus CalmetteGuréin vaccination has been mandatory since 1953 in South Korea (21). All miliary TB cases (aged $\geq 18$ years) attending the hospital from August, 2001 to September, 2016 were selected using ICD-10 code A190-A199. Those with available chest radiographs and CT scans performed on admission were enrolled and their medical records were reviewed. Patients with human immunodeficiency virus (HIV) infection were excluded. The eligible patients were categorized into the three groups according to the classification adopted in a previous study (22): (I) young adult group (18-40 years); (II) middle-aged adult group (41-64 years); (III) old adult group ( $\geq 65$ years). Details regarding patient demographics, clinical manifestations (including presenting symptoms and laboratory, microbiological, and radiological findings), clinical course, treatment outcome, and the cause of death were obtained from the medical records. A standard regimen consisting of isoniazid, rifampicin, ethambutol, and pyrazinamide was administered during the intensive phase, as recommended by the National Tuberculosis Program (23), unless drug modification was necessary due to adverse drug reactions or drug resistance. The study was approved by the Institutional Review Board (IRB) of the Kyungpook
National University Hospital (IRB No. 2017-05-018). Informed consent was waived by the IRB.

\section{Diagnostic criteria and definition}

The diagnosis of miliary $\mathrm{TB}$ in patients showing miliary patterns on radiological examinations, was made when one of the following was met: (I) mycobacterial evidence of infection with MTB, such as positive acid-fast bacilli (AFB) smear, TB-polymerase chain reaction (TB-PCR), or culture from sputum, bronchial aspirate, urine, body fluid, or tissue; (II) the presence of chronic granulomatous inflammation in tissue, and clinical and radiographic improvement with anti-TB drug medication. Underlying chronic condition included malignancy, transplantation, end-stage renal disease, advanced liver cirrhosis, immunosuppressive therapy, diabetes mellitus, previous gastrectomy, or chronic heart, lung, or neurological disease.

A "typical" miliary pattern on chest X-ray was defined as a collection of tiny discrete pulmonary opacities that were generally uniform in size and widespread in distribution, each of which measured $\leq 3 \mathrm{~mm}$ in diameter (1). An "atypical" miliary pattern was defined as the obscuring of background miliary nodules by diffuse ground glass opacity (GGO) or the other findings (1). On chest CT, recognizable miliary nodules were defined when randomly distributed micro-nodules were well discernible (24). Chest radiographs and CT were reviewed by a board-certified radiologist and a pulmonologist. If a discrepancy was noted between investigators, the images were further reviewed by another pulmonologist who was blinded to the results.

Treatment outcome category was defined according to the revised 2013 World Health Organization (WHO) definition (25). In the specific causes of death, TB-related septic shock was defined as hypotension persisting despite adequate fluid resuscitation during the intensive phase of anti-TB treatment, which was induced by TB without other etiology of infection, and TB-unrelated bacterial septic shock as that due to other new bacterial infection in the absence of TB progression throughout the anti-TB treatment.

\section{Statistical analysis}

Statistical analyses were performed using IBM SPSS Statistics for Windows, Version 22.0 (IBM Corp., Armonk, NY, USA). Continuous variables were expressed as the median (interquartile range [IQR]) and differences between groups were analyzed using the Mann-Whitney test. 
Categorical variables were expressed as absolute values and percentages, and were analyzed using the Chi square or Fisher's exact tests. All-cause and TB-related mortality rates were compared between the age groups by KaplanMeier survival curve. To identify independent predictor for TB-related deaths, Cox's proportional hazards analysis was employed. Variables with $\mathrm{P}<0.05$ in univariate analysis were entered into the multivariate regression analysis with the backward elimination method. A $\mathrm{P}<0.05$ was considered significant.

\section{Results}

\section{Demographics}

Among the 260,001 patients hospitalized with active TB during the time of the study, a total of 160 adult patients diagnosed with miliary TB were identified. Of these, 132 patients had positive MTB culture from various specimens [sputum $(n=92)$, bronchial aspirate $(n=52)$, urine $(n=15)$, and pleural fluid or tissue ( $\mathrm{n}=9)$ ], 15 patients harbored histologically chronic granulomatous inflammation with additional positive TB-PCR $(n=6)$ or AFB smear $(n=2)$ in tissue, and 3 patients had a positive sputum AFB smear $(\mathrm{n}=1)$, positive bronchial aspirate AFB smear and TB-PCR $(\mathrm{n}=1)$, and positive CSF TB-PCR $(\mathrm{n}=1)$. Eight patients were excluded because they did not meet microbiological or histological criteria, and two were excluded due to HIV co-infection. Thus, 150 adult patients were included in the final analysis. In terms of socioeconomic status, there were $18(12 \%)$ patients with the lowest income who acquired medical aid from the national government. There were no cases of drug addiction, but one patient with liver cirrhosis was addicted to alcohol. The numbers of cases in every 5 -year period since 2001 were 34,41 , and 75 cases, respectively.

Overall, $71(47 \%)$ patients were male and the median age was 68 years (range 18-91 years). According to the classification by age range, 27,35 , and 88 patients composed the young, middle-aged, and old adult groups, respectively (Table 1). The frequency of underlying chronic condition was significantly greater in the old adult group than in the young and middle-aged groups (49\% vs. $26 \%$ and $29 \%$, respectively; $\mathrm{P}<0.05)$.

\section{Clinical manifestations}

Fever was the most frequent sign in all adult age groups.
However, presenting sign or symptoms such as fever, dyspnea, and weight loss were not significantly different among the three groups. The median duration of illness prior to hospitalization was 30 days in each group.

Anemia was common (60-70\%) and C-reactive protein (CRP) levels were moderately elevated $(6.4-7.7 \mathrm{mg} / \mathrm{dL})$ in all three groups. Serum albumin levels in the old adult group were the lowest and significantly different compared to those of the young adult group ( 2.8 vs. $3.4 \mathrm{~g} / \mathrm{dL}, \mathrm{P}<0.05)$. However, the frequency of anemia and hyponatremia, and laboratory parameters, including white blood cells, hemoglobin levels, and CRP levels, were not significantly different among the three groups. Sputum AFB smear and culture results were comparable among all groups.

Typical miliary patterns on chest X-ray were more frequent in the young adult group than in the old adult group $(67 \%$ vs. $47 \%, \mathrm{P}<0.05)$. However, identification of miliary nodules on chest CT was not significantly different among the groups. GGO on chest CT was significantly greater in the old group than in the young group $(78 \% v s$. $56 \%, \mathrm{P}<0.05)$, whereas mediastinal lymphadenopathy was more frequent in the young group than in the old group ( $41 \%$ vs. $19 \%, \mathrm{P}<0.05$ ). Preexisting active and cavitary lung lesions were not significantly different among the three groups.

\section{Clinical course and treatment outcome}

The time to anti-TB therapy with a median time of 1 day was the same in each of the three groups (Table 2). Inhospital mortality and hospital length of stay were not significantly different among the groups. However, the completion of successful anti-TB therapy was significantly higher in the young adult group than in the old group ( $89 \%$ vs. $56 \%, \mathrm{P}<0.05$ ). This finding was attributable to the greater frequency of overall deaths during anti-TB therapy in the old adult group $(n=27)$ than in the young group $(n=2)$ ( $31 \%$ vs. $7 \%, \mathrm{P}<0.05$ ). Additionally, the cause of death was significantly different among the groups $(\mathrm{P}=0.008)$. All of the deaths in the young $(n=2)$ and middle-aged $(n=6)$ adult groups were directly related to miliary $\mathrm{TB}$, whereas deaths in the old group were more attributable to TB-unrelated deaths $(n=16)$ rather than TB-related deaths $(n=11)$. Thus, TB-related deaths were not significantly different among the groups $(\mathrm{P}=0.512)$, although all-cause deaths were significantly different among the groups $(\mathrm{P}=0.028)$ (Figure 1).

Figure 2 shows the specific cause of TB-related and TBunrelated deaths in the old adult group. With respect to 
Table 1 Demographic, clinical, laboratory, and radiological findings according to age groups in adult patients with miliary tuberculosis

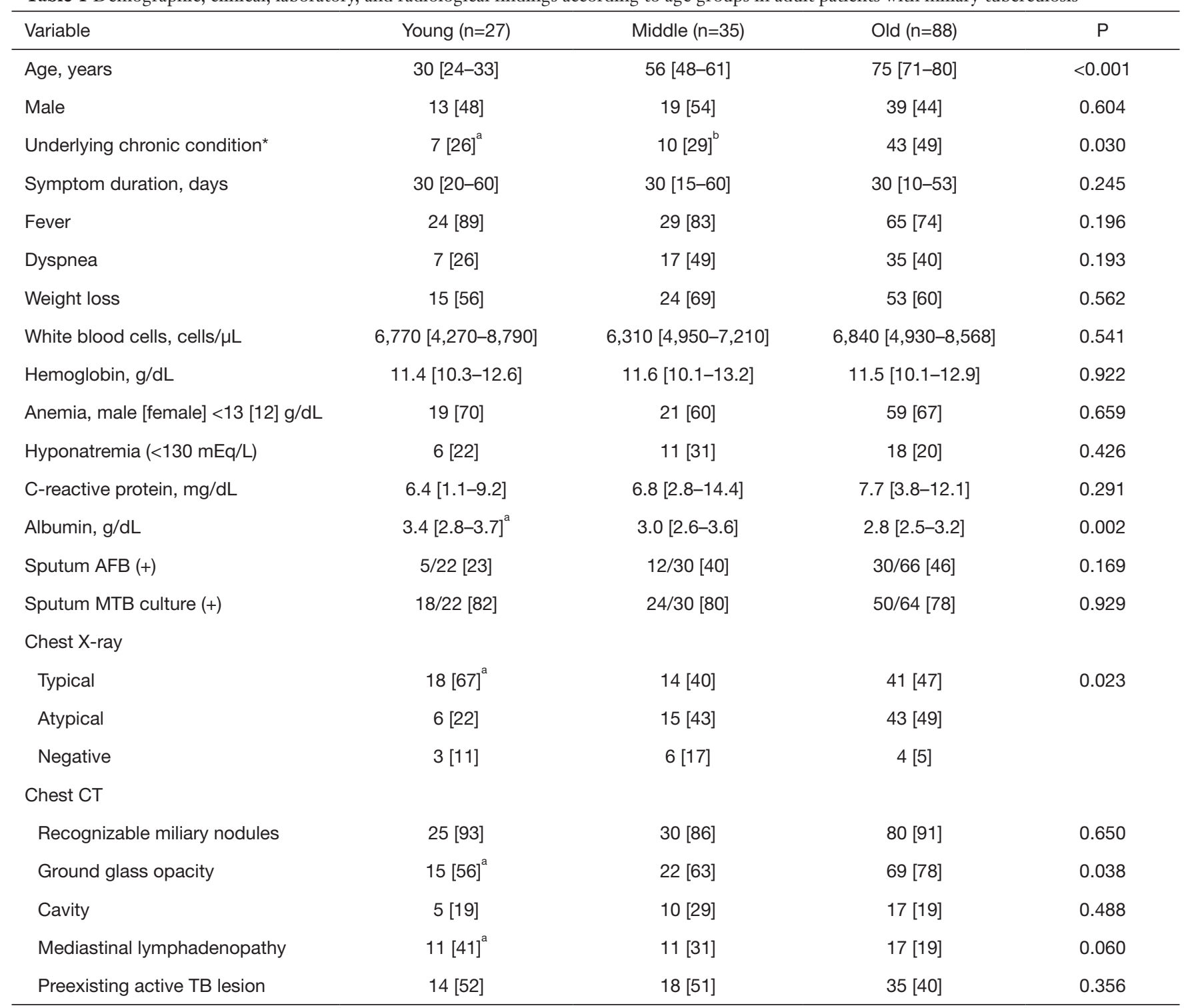

Data are presented as the number [\%] or median [IQR]. *, Includes patients with malignancy, transplantation, end-stage renal disease, advanced liver cirrhosis, immunosuppressive therapy, diabetes mellitus, gastrectomy, or chronic heart, lung, or neurological disease. a $\mathrm{P}<0.05$, comparison between the young and old adult groups. ${ }^{\mathrm{b}}, \mathrm{P}<0.05$, comparison between the middle-aged and old adult groups. AFB, acid fast bacilli; MTB, Mycobacterium tuberculosis; CT, computed tomography; TB, tuberculosis.

TB-related deaths, the most frequent cause was respiratory failure $(n=6)$, followed by TB-related septic shock $(n=3)$, TB encephalitis $(n=1)$, and anti-TB drug-induced pseudomembranous colitis and erythema multiforme $(n=1)$. All but two deaths (one TB encephalitis and one anti-TB drug-induced pseudomembranous colitis and erythema multiforme) occurred during the intensive phase. In TBunrelated deaths, cardiovascular disease $(n=7)$ was the most frequent cause, followed by malignancy $(n=4)$, bacterial septic shock $(n=2)$, subdural hemorrhage $(n=1)$, and unknown $(\mathrm{n}=2)$. Three patients (two cardiovascular diseases and one malignancy) died in the intensive phase, whereas the remaining 13 patients died in the continuation phase. One patient with unknown cause of death had underlying diabetes mellitus and the other had chronic heart and neurological disease. The deaths in these two patients occurred without the aggravation of respiratory symptoms and chest radiograph after 4 months of anti-TB treatment. 
Table 2 Clinical course, treatment outcome, and cause of death according to age groups in adult patients with miliary tuberculosis

\begin{tabular}{|c|c|c|c|c|}
\hline Variable & Young $(n=27)$ & Middle $(n=35)$ & Old $(n=88)$ & $P$ \\
\hline In-hospital mortality & $2[7]$ & $5[14]$ & $9[10]$ & 0.711 \\
\hline Hospital length of stay, days & $13[6-26]$ & 15 [9-26] & $16[11-23]$ & 0.586 \\
\hline \multicolumn{5}{|l|}{ Treatment outcome } \\
\hline Treatment incomplete & $3[11]$ & 10 [29] & 39 [44] & 0.004 \\
\hline Lost to follow-up & $1[4]$ & $4[11]$ & $10[11]$ & 0.563 \\
\hline Transfer & 0 & 0 & 2 [2] & 1.0 \\
\hline Died & $2[7]^{a}$ & $6[17]$ & 27 [31] & 0.027 \\
\hline TB-unrelated & - & - & $16[59]$ & 0.008 \\
\hline
\end{tabular}

Data are presented as the number [\%] or median [IQR]. ${ }^{a}, \mathrm{P}<0.05$, comparison between the young and old adult groups; ${ }^{\mathrm{b}}$, $\mathrm{P}<0.05$, comparison between the middle-aged and old adult groups. TB, tuberculosis.
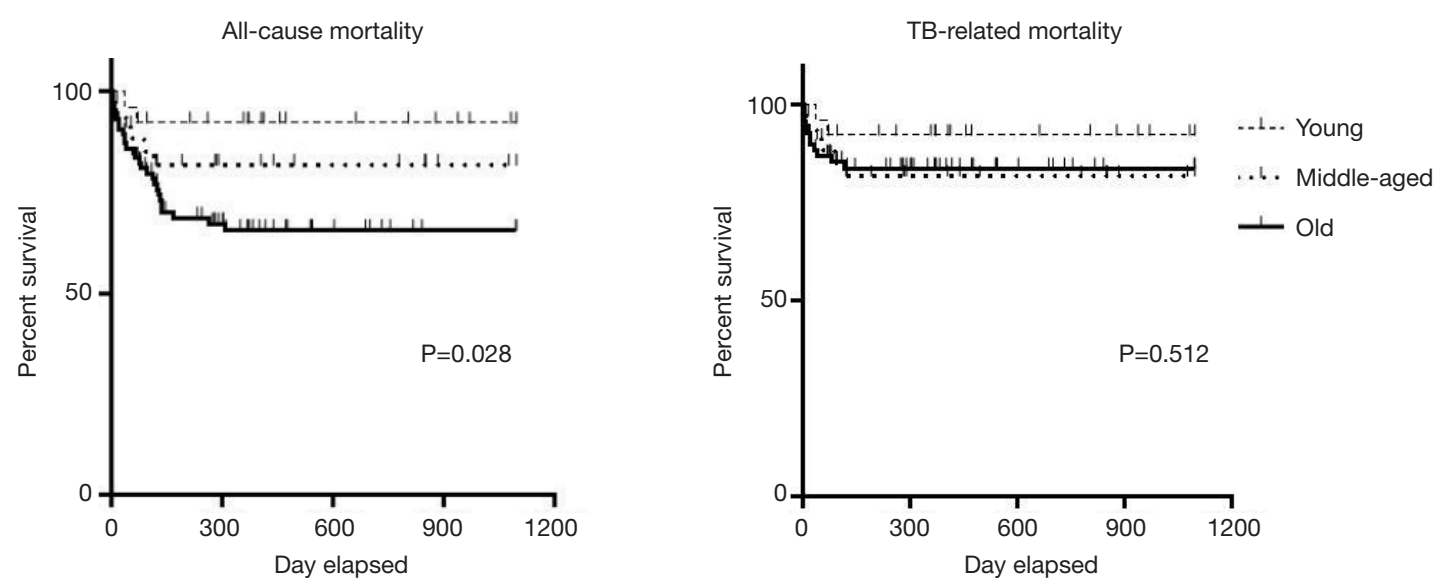

Figure 1 Kaplan-Meier survival curve according to different age groups in adult patients with miliary tuberculosis. Young adults: 18-40 years; Middle-aged adults: 41-64 years; Old adults: $\geq 65$ years. TB, tuberculosis.

All TB-related deaths in the young and middle-aged adult groups were due to acute respiratory failure $(\mathrm{n}=5)$, TB-related septic shock $(\mathrm{n}=2)$, and anti-TB drug-induced hepatic failure $(\mathrm{n}=1)$.

\section{Risk factors for TB-related deaths}

Risk factors for TB-related deaths were determined in the combined group of young and middle-aged adult ( $<65$ years) and the old adult group ( $\geq 65$ years) (Table 3 ).
In univariate Cox regression analysis, underlying chronic condition, lower hemoglobin levels, increased CRP levels, lower albumin levels, and acute respiratory failure were risk factors for TB-related mortality in the combined young and middle-aged group. In the old adult group, increasing age, increased CRP levels, lower albumin levels, and acute respiratory failure were predictors for TB-related deaths. The multivariate Cox regression analysis revealed that underlying chronic condition, lower hemoglobin levels, and acute respiratory failure were independent predictors for 

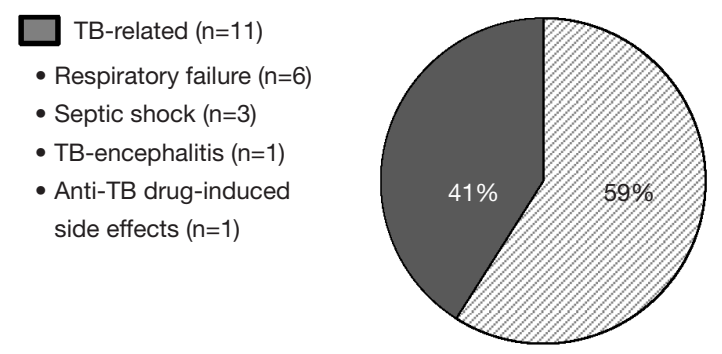

$\mathscr{Z}$ TB-unrelated $(n=16)$

- Cardiovascular disease $(n=7)$

- Malignancy $(n=4)$

- Bacterial septic shock $(n=2)$

- Subdural hemorrhage $(n=1)$

- Unknown $(n=2)$

Figure 2 Specific cause of tuberculosis (TB)-related and TB-unrelated deaths in old adult patients with miliary tuberculosis who died during anti-tuberculosis treatment.

Table 3 Risk factors for TB-related deaths in young-middle and old adult patients with miliary tuberculosis

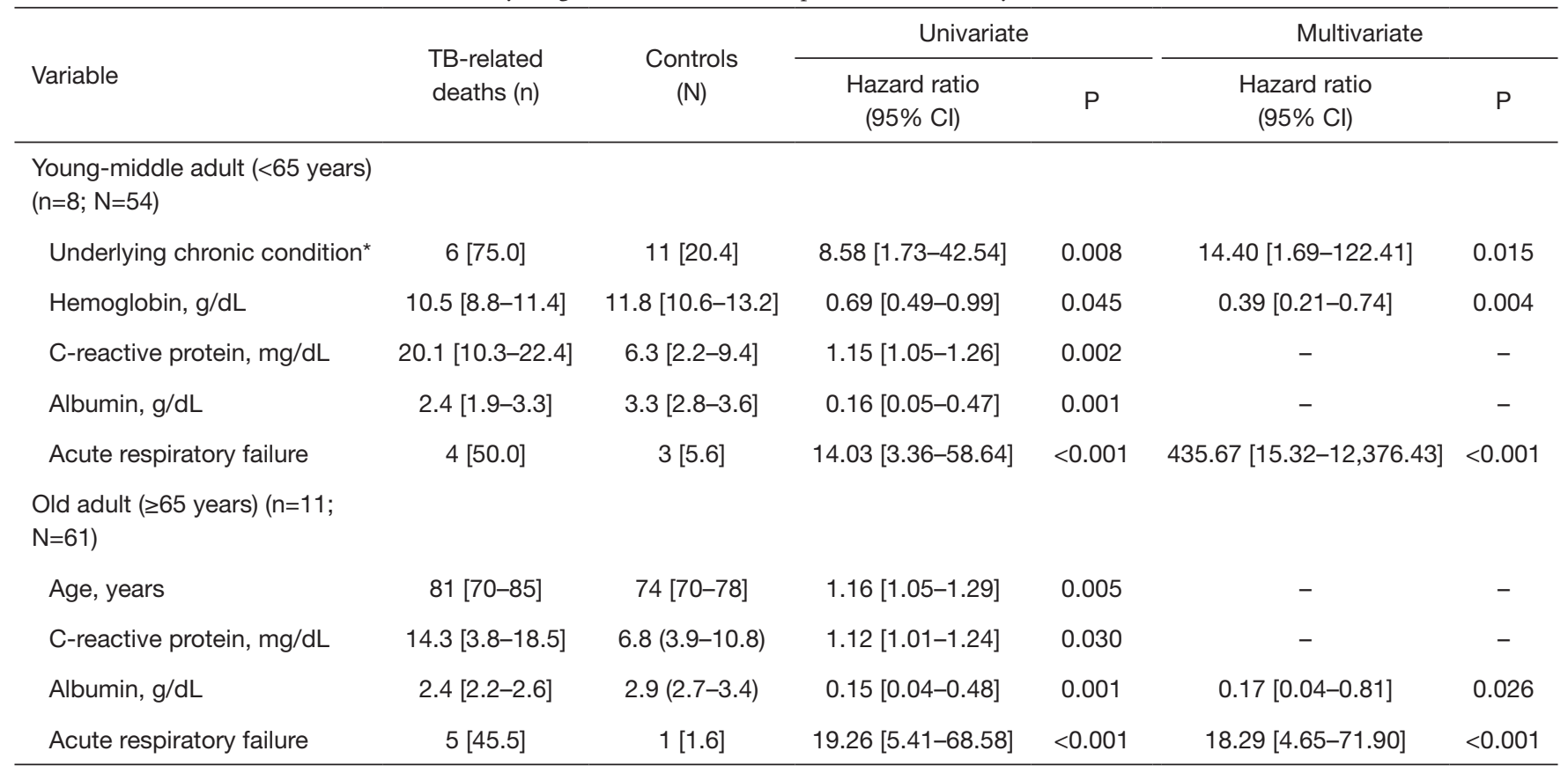

Data are presented as the number [\%] or median [IQR]. *, Includes patients with malignancy, transplantation, end-stage renal disease, advanced liver cirrhosis, immunosuppressive therapy, diabetes mellitus, gastrectomy, or chronic heart, lung, or neurological disease. TB, tuberculosis; $\mathrm{Cl}$, confidence interval.

mortality in the combined young and middle-aged adult group ( $<65$ years), and lower albumin levels and acute respiratory failure were those in the old adult group ( $\geq 65$ years).

\section{Discussion}

The present study classified adult patients with miliary TB into three groups according to age range. The main findings were as follows: (I) overall clinical manifestations were not significantly different among the three groups;
(II) treatment completion rate was significantly lower and overall deaths were significantly higher in the old adult group than in the young adult group; (III) however, the cause of deaths in the young and middle-aged group were all TB-related, whereas more than half of the deaths in the old group was not directly related to TB. Thus, TBrelated deaths were not significantly different among the groups; (IV) in multivariate analysis, underlying chronic condition, lower hemoglobin levels, and acute respiratory failure were independent predictors for TB-related death in the combined group of young and middle-aged adults 
(<65 years), and lower albumin levels and acute respiratory failure were those in the old adult group ( $\geq 65$ years).

Although the incidence of TB has been slowly decreasing with the implementation of TB control programs in Korea (20), the current study showed that miliary TB was more prevalent in the recent 5-year period than in past 5 -year periods. These findings suggest that the incidence of miliary TB may relatively increase with increasing life expectancy, co-morbidity, and widespread use of immunosuppressive drugs. The sex distribution was similar overall and was not significantly different between the three adult groups. However, women seem to be somewhat more frequent in the old adult group (Table 1), as shown in other adult series $(17,18)$ after 2000 .

Clinical and laboratory findings were frequently abnormal, as in previous reports (9-19), but were not unique to the specific age group. However, the presence of fever, which is the most important clinical sign in miliary TB $(1,19)$, was less frequent according to increasing age groups (89\%, 83\%, and $74 \%$ in the young, middle-aged, and old adult groups, respectively), although statistical significance between the groups was not achieved. In addition, the sensitivity of sputum culture for MTB was quite similar (approximately $80 \%$ ), but the sputum for examination was less available in the old adult group.

Radiological findings provide valuable diagnostic clue in miliary TB. The diagnostic sensitivity of chest $\mathrm{X}$-ray showed significant difference between the young and old adult groups (67\% vs. $47 \%$, respectively), although the overall diagnostic sensitivity of chest X-ray was in line with that demonstrated in previous studies (1-3). In contrast, chest CT revealed miliary nodules in approximately $90 \%$ of all adult groups. This finding suggests that the diagnosis of miliary TB has been greatly improved by the application of chest CT than in the past. The greater frequency of GGO on chest CT in the old adult group may lead to a lower frequency of typical finding on chest X-ray in the old adult group (24). A few patients without recognizable miliary patterns on chest CT showed miliary nodules on short-term follow-up chest radiographs or CT scans taken after the GGO finding had resolved.

Overall TB deaths, which are defined as deaths from any cause occurring during anti-TB treatment according to the WHO definition (25), were 23\% (35/150). This mortality rate falls within the mortality range in previous reports (9-19). However, the mortality rate was significantly different between the young and old adult group in our study. This finding is supported by two previous studies, which showed considerable difference between the two groups at a cut-off of 40 years $(4,14)$. It has been frequently reported that the mortality has remained high, despite the introduction of rifampicin in the 1970s $(9,10)$ and available chest CT in the 1990s (17-19). These persistent high overall mortality rates may be partly explained by the increasing proportion of elderly patients with higher mortality.

Of the 35 overall TB deaths, 19 (54\%) deaths were directly related to TB. TB-related death was attributable to more severe form of miliary $\mathrm{TB}$, which implies delayed diagnosis and treatment. Anti-TB treatment after presentation to the hospital in our study was not delayed in all age groups (median 1 day). This finding suggests that there is a delay in seeking medical attention at a hospital or a delay in diagnosis before transfer to tertiary referral hospital, which should be improved to further reduce TBrelated deaths.

In contrast to TB-related deaths, TB-unrelated deaths have received little attention in most previous reports. In our study, these deaths occurred only in the old age group, and contributed to the high overall mortality and significant difference in mortality between the young and old adult groups, as well as in the cause of death according to age groups. Most of the deaths occurred in relatively stable status during the continuation phase of treatment (median 116 days). The old age group commonly had other coexisting diseases that may have had a significant impact on prognosis. Furthermore, 14 (88\%) of the 16 patients with TB-unrelated death in our study had other underlying condition. Advanced age and underlying comorbid illnesses are well-known risk factors for overall TB deaths (26); these are unavoidable factors. However, clinical symptoms of miliary TB may be shared with other diseases. Particularly, comorbidities with symptoms overlapping with those of miliary TB, such as dyspnea in cardiovascular disease, may be overlooked. In addition, anti-TB drugs, especially rifampicin, may adversely affect treatment of other disorders. Thus, our results suggest that the management of elderly patients with miliary TB should include identification and treatment of their comorbidities.

It may be clinically more practical to identify risk factors for TB-related deaths according to age groups than in overall adults with a wide age range. The main findings of risk factors for TB-related deaths in the adult patients $<65$ and $\geq 65$ years, respectively, represented the advanced stage of miliary TB. These risk factors were also separately identified in previous studies $(3,5,10,13,18,19)$. It is notable that increasing age, which was most frequently observed as 
an independent predictor for poor outcome $(4,5,10,11,19)$, did not independently correlate with TB-related deaths, when adult patients were categorized as younger or older based on the age of 65 years. However, a few TB-related deaths in our study may be insufficient to determine the independent risk factors for mortality in each group. Further study with a large population is warranted to identify risk factors according to age groups.

The main limitation of our study is the retrospective design, as with most of the other studies. Some patients with miliary TB were clinically diagnosed. These patients were excluded due to the strict inclusion criteria in the present study. So, there may be selection bias and the overall mortality rate according to age groups may have been underestimated. Second, in our study population, a variety of diseases responsible for miliary patterns on chest radiology were not completely assessed due to the study's retrospective design. However, simultaneous occurrence of miliary TB and other diseases is not likely to be common. Third, the sample size included in each group was relatively small, particularly for the analysis of some factors, as mentioned above. Lastly, there were a few patients lost to followup. Thus, we cannot exclude the effect of these patients. However, it is unlikely that the treatment outcome of the old adult patients lost to follow-up was better than that of the young or middle-aged adult patients lost to follow-up.

In conclusion, the present study showed different treatment outcomes associated with the cause of death according to age groups in adult patients with miliary TB. In addition, our data suggest that the independent risk factors for TB-related deaths may be different between the age groups.

\section{Acknowledgements}

Funding: This work was supported by the National Research Foundation of Korea grant funded by the Korean government (2016R1C1A2A01052502).

\section{Footnote}

Conflict of Interest: The authors have no conflicts of interest to declare.

Ethical Statement: The study was approved by the Institutional Review Board (IRB) of the Kyungpook National University Hospital (IRB No. 2017-05-018). Informed consent was waived by the IRB.

\section{References}

1. Sharma SK, Mohan A, Sharma A, et al. Miliary tuberculosis: new insights into an old disease. Lancet Infect Dis 2005;5:415-30.

2. Slavin RE, Walsh TJ, Pollack AD. Late generalized tuberculosis: a clinical pathologic analysis and comparison of 100 cases in the preantibiotic and antibiotic eras. Medicine (Baltimore) 1980;59:352-66.

3. Munt PW. Miliary tuberculosis in the chemotherapy era: with a clinical review in 69 American adults. Medicine (Baltimore) 1972;51:139-55.

4. Gelb AF, Leffler C, Brewin A, et al. Miliary tuberculosis. Am Rev Respir Dis 1973;108:1327-33.

5. Grieco $\mathrm{MH}, \mathrm{Chmel} \mathrm{H}$. Acute disseminated tuberculosis as a diagnostic problem. A clinical study based on twentyeight cases. Am Rev Respir Dis 1974;109:554-60.

6. McGuinness G, Naidich DP, Jagirdar J, et al. High resolution CT findings in miliary lung disease. J Comput Assist Tomogr 1992;16:384-90.

7. Oh YW, Kim YH, Lee NJ, et al. High-resolution CT appearance of miliary tuberculosis. J Comput Assist Tomogr 1994;18:862-6.

8. Hong SH, Im JG, Lee JS, et al. High resolution CT findings of miliary tuberculosis. J Comput Assist Tomogr 1998;22:220-4.

9. Kim JH, Langston AA, Gallis HA. Miliary tuberculosis: epidemiology, clinical manifestations, diagnosis, and outcome. Rev Infect Dis 1990;12:583-90.

10. Maartens G, Willcox PA, Benatar SR. Miliary tuberculosis: rapid diagnosis, hematologic abnormalities, and outcome in 109 treated adults. Am J Med 1990;89:291-6.

11. Sime PJ, Chilvers ER, Leitch AG. Miliary tuberculosis in Edinburgh--a comparison between 1984-1992 and 19541967. Respir Med 1994;88:609-11.

12. Ormerod LP, Horsfield N. Miliary tuberculosis in a high prevalence area of the U.K.: Blackburn 1978-1993. Respir Med 1995;89:555-7.

13. Sharma SK, Mohan A, Pande JN, et al. Clinical profile, laboratory characteristics and outcome in miliary tuberculosis. QJM 1995;88:29-37.

14. Long R, O'Connor R, Palayew M, et al. Disseminated tuberculosis with and without a miliary pattern on chest radiograph: a clinical-pathologic-radiologic correlation. Int J Tuberc Lung Dis 1997;1:52-8.

15. Al-Jahdali H, Al-Zahrani K, Amene P, et al. Clinical aspects of miliary tuberculosis in Saudi adults. Int J Tuberc Lung Dis 2000;4:252-5. 
16. Alsoub H, Al Alousi FS. Miliary tuberculosis in Qatar: a review of 32 adult cases. Ann Saudi Med 2001;21:16-20.

17. Mert A, Bilir M, Tabak F, et al. Miliary tuberculosis: clinical manifestations, diagnosis and outcome in 38 adults. Respirology 2001;6:217-24.

18. Hussain SF, Irfan M, Abbasi M, et al. Clinical characteristics of 110 miliary tuberculosis patients from a low HIV prevalence country. Int J Tuberc Lung Dis 2004;8:493-9.

19. Mert A, Arslan F, Kuyucu T, et al. Miliary tuberculosis: Epidemiologicaland clinical analysis of large-case series from moderate to low tuberculosis endemic Country. Medicine (Baltimore) 2017;96:e5875.

20. World Health Organization. Global tuberculosis report 2015 [Internet]. Available online: http://www.who.int/tb/ data [accessed on 9 February 2017].

21. Joung SM, Ryoo S. BCG vaccine in Korea. Clin Exp Vaccine Res 2013;2:83-91.

Cite this article as: Lee J, Lim JK, Kim EJ, Lee DH, Kim YK, Yoo SS, Lee SY, Cha SI, Park JY, Kim CH. Comparison of clinical manifestations and treatment outcome according to age groups in adult patients with miliary tuberculosis. J Thorac Dis 2018;10(5):2881-2889. doi: 10.21037/jtd.2018.04.139
22. Chung WS, Lin CL, Hung CT, et al. Tuberculosis increases the subsequent risk of acute coronary syndrome: a nationwide population-based cohort study. Int J Tuberc Lung Dis 2014;18:79-83.

23. Joint Committee for the Development of Koran Guidelines for Tuberculosis Korean Centers for Disease Control and Prevention. Korean Guidelines for Tuberculosis, Second Edition, 2014. Available online: http://www.lungkorea.org/thesis/file/korean_guidelines_ for tuberculosis_2014.pdf

24. Lee J, Lim JK, Seo H, et al. Clinical relevance of ground glass opacity in 105 patients with miliary tuberculosis. Respir Med 2014;108:924-30.

25. WHO revised definitions and reporting framework for tuberculosis. Euro Surveill 2013;18:20455.

26. Waitt CJ, Squire SB. A systematic review of risk factors for death in adults during and after tuberculosis treatment. Int J Tuberc Lung Dis 2011;15:871-85. 